\title{
Curiosity, Inquiry and the Geographical Imagination
}

\author{
A. Haller \\ Institut für Geographie, Universität Innsbruck, Innrain 52f, 6020 Innsbruck, Austria
}

Correspondence to: A. Haller (andreas.haller@uibk.ac.at)

Gade, D. W.: Curiosity, Inquiry and the Geographical Imagination, New York, Peter Lang Publishing, 307 pp., ISBN-13: 9781433115417 (Hardcover), €41.40, 2011.

„Man kann einem Menschen nichts lehren, man kann ihm nur helfen, es in sich selbst zu entdecken“ (Galileo Galilei)

Das vorliegende, in englischer Sprache verfasste Buch von Daniel W. Gade stellt inhaltlich auf den ersten Blick eine Rarität dar; während ein Großteil wissenschaftlicher Publikationen die Ergebnisse getaner Arbeit präsentiert, so befasst sich diese Monographie mit der Frage, welche persönlichen Motivationen hinter den Forschungstätigkeiten stehen. Für den in erster Linie durch die Berkely School (Carl O. Sauer, James J. Parsons, William M. Denevan) sozialisierten Autor sind die menschliche Wissbegierde (epistemic curiosity) sowie die Liebe zum Forschungsgegenstand (romantic sensibility) dabei die bedeutendsten Triebfedern für die Erlangung neuer Erkenntnisse im Grenzbereich zwischen Geographie, Geschichtswissenschaft und Ethnologie. Seine Sicht der Dinge - welche an ein Zitat erinnert das dem toskanischen Gelehrten Galileo Galilei zugeschrieben wird - versucht der Autor durch die Interpretation ausgewählter Biographien (besonders jener von Sauer) sowie persönlicher Introspektion und Retrospektive dem Leser verständlich und nachvollziehbar zu vermitteln. Konkret nennt Gade neben dem eingangs genannten Ziel der Arbeit noch drei weitere Absichten; erstens soll Forschung als ein kontinuierlicher Prozess dargestellt werden, welcher mit Ideen im Kopf des Einzelnen beginnt und schließlich als geschriebenes Werk sein - vorläufiges Ende findet. Zweitens möchte der Autor thematisieren, wie Leben und Wissenschaft im Goethe'schen Sinne (,Überall lernt man nur von dem, den man liebt") untrennbar miteinander in Verbindung stehen. Drittens will Gade den Charakter und die spezielle Perspektive der Sauer'schen culturalhistorical geography - mit welcher auch einige deutschsprachige Geographen durch Professuren oder Gastprofessuren (z. B. Oskar Schmieder, Gottfried Pfeifer oder Herbert Wil- helmy) in Berkeley im Austausch standen (Leighly, 1979; Borsdorf und Leser, 2003) - beschreiben und erklären.

Die Publikation gliedert sich folglich in drei Teile. In Part One: Curiosity in a Broad Context widmet sich Gade zu Beginn dem Phänomen der Wissbegierde in allgemeiner Sicht - und spannt dabei den zeitlichen Bogen von den Anfängen der Menschheit über die Renaissance hin zur Gegenwart; am Beispiel von westlichen und nicht-westlichen Kulturkreisen versucht der Autor den Stellenwert von Neugier und Wissbegierde - sowie dessen Auswirkungen auf die Entwicklung der Gesellschaften - in unterschiedlichen Epochen aufzuzeigen. Nach dieser, etwas weit ausholenden und - sinnvollerweise - oberflächlichen Einleitung und Begriffserklärung wird der Leser zu konkreten Persönlichkeiten der Geschichte, deren Leben von Wissbegierde geprägt wurde, hingeführt. Die interessante Auswahl dieser - durchwegs männlichen Kurzbiographien reicht dabei von anglophonen (z. B. Thomas Jefferson oder John K. Wright) über deutschsprachige Autoren (z. B. Alexander von Humboldt, Fritz Müller oder Erwin H. Ackerknecht) bis hin in den romanischen Sprachraum (z. B. Marcos Jiménez de la Espada, Mosè Bertoni oder Jean-Jacques Rousseau), und lässt Rückschlüsse auf die besondere Sprachgewandtheit des Verfassers zu. In einem abschließenden Vergleich, zeichnet Gade trotz vieler Gemeinsamkeiten ein differenziertes Bild der von innerer Wissbegierde getriebenen Persönlichkeiten - von aufklärerisch denkenden hin zu romantischen Charakteren. In einem gut strukturierten, abschließenden Kapitel des ersten Teils zieht Gade den Vergleich zwischen Wissenschaftlern und Schamanen; anhand einer nachvollziehbaren Analyse von Aufgaben, Lebensweisen sowie der Art zu Denken weist er abschließend zu Recht darauf hin, dass der Archetypus des von Wissbegierde getriebene Menschen, welcher in allen gesellschaftlichen Gruppen (unabhängig von Ethnizität, Geschlecht oder Religion) existiert, nicht nur im akademischen Kontext vorkommt: „Universities, institutes, laboratories, foundations and publication programs are the results, not the causes, of 
the human compulsion to explore the borders of the known" (S. 49).

Mit einem treffenden Zitat von Friedrich Freiherr von Hardenberg alias Novalis („Das Herz ist der Schlüssel der Welt und des Lebens") beginnt Daniel W. Gade das erste Kapitel von Part Two: Curiosity in Disciplinary Framework - treffend deshalb, weil die romantische (gegenaufklärerische) Wissenschaft, und mit ihr der Einfluss deutschsprachiger Denker, den roten Faden auf dem Streifzug durch die US-amerikanische Geographie Sauer'scher Prägung darstellen. Zu Beginn des zweiten Teils führt Gade in die von innerer Wissbegierde des Individuums getriebene romantische Wissenschaft ein, welche - nach seiner Auffassung nicht auf die Epoche der Romantik beschränkt ist, sondern sowohl vor (z. B. Giambattista Vico), als auch nach (z. B. Hans-Georg Gadamer) diesem Zeitabschnitt existiert. Im Anschluss wird - hinsichtlich der Geographie - die europäische Forschungstradition eines Friedrich Ratzel oder Paul Vidal de la Blache kurz erwähnt, da der Autor diese als bedeutsam für die Entwicklung der Sauer'schen Geographie romantischer Prägung (cultural-historical geography) ansieht. In einem kurzen Vergleich wird letztere der humanistischen Geographie eines John K. Wright oder Yi-Fu Tuan gegenübergestellt und betont, dass trotz vieler Gemeinsamkeiten einige epistemologische Unterschiede existieren - eine Ansicht die auch Tuan selbst zu bestätigen scheint (Tuan, 2012:17). Es folgt eine hervorragend strukturierte und prägnante $\mathrm{Be}$ schreibung der Eigenschaften Sauer'scher Geographie (z. B. der Historismus oder historicism, die Suche nach dem Exotischen, die idiographische und individualistische Arbeitsweise sowie das Interesse an der Gestalt von - holistisch betrachteten - Kulturlandschaften), welche unverkennbar Parallelen zur traditionellen Länder- und Landschaftskunde des deutschsprachigen Raums aufweisen. In Sauers Begeisterung für das Humboldt'sche Bildungsideal und die Einheit von Forschung und Lehre sieht Daniel W. Gade schließlich gewichtige Gründe für die wissenschaftlichen Erfolge der ersten Generation von Sauerians, die in der Meinung des Autors von innerer Wissbegierde angetrieben - und ohne soziale Verpflichtungen - ihrem Herzen folgten (S. 136). Trotz seiner eher idealistischen Ansichten ist sich der Autor jedoch bewusst, dass heutzutage die Wege jüngerer Fachkollegen - obwohl häufig von Wissbegierde angetrieben - zunehmend durch die „gesellschaftliche Relevanz“ des Forschungsthemas und die Erhältlichkeit von Drittmitteln (welche wiederum von Modetrends beeinflusst ist) gelenkt werden: „Curiosity-driven scholarship conflicts with agendas now dominating many institutions" (S. 157).

Mit Part Three: Curiosity in Reflexive Mode beleuchtet der Autor seine eigenen Erfahrung aus fünf Jahrzehnten Forschungstätigkeit, und informiert den Leser in unterhaltsamer Art und Weise über Projektideen, welche bisher noch nicht umgesetzt wurden. In Form eines autobiographischen Rückblicks analysiert Gade seine wissenschaftliche Arbeitsweise anhand von einigen Eigenschaften, die sich wie ein ro- ter Faden durch die letzten fünf Jahrzehnte ziehen. Erstens, der Wunsch neues zu erfahren und zu lernen; zweitens, seine Suche nach Authentizität, Ästhetik und dem Unvollkommenen; drittens, eine bestimmte Art des Forschungsprozesses, die besonders auf der eigenen Vorstellungskraft, Büchern und Bibliotheken aufbaut, um schließlich über intensive, von Selbstreflexion begleitete Feldarbeit in eine Schreibphase zu münden. Die Präferenz des Autors für das geschriebene Wort - und seine geringe Begeisterung für mündliche Vorträge ist dabei klar herauszulesen. Dies wird auch in seiner Meinung über book reviews klar, welche für ihn wertvolle, intellektuelle Reisen in das Innere eines Werkes darstellen. Anhand von ausgewählten Themen, die die Neugier von Daniel W. Gade geweckt haben - jedoch noch nicht in die Schreibphase gelangten - beschreibt er deren Entstehungsgeschichte und Kontext. Ergänzend erwähnt er jedoch auch Beispiele, die zu Beginn viel versprechend waren - um letztlich im Nichts zu enden. Es sind Abschnitte wie diese, die die vorliegende Publikation inhaltlich besonders lesenswert machen, da sie Situationen behandeln, die wohl jeder wissenschaftlich Tätige schon erfahren hat - geschrieben wurde darüber bisher allerdings kaum.

Anstatt die Ergebnisse von fünf Jahrzehnten geographischer Arbeit zusammenzufassen, legt Daniel W. Gade mit dieser Monographie ein vom Anfang bis zum Schluss stringentes und stilistisch anspruchsvolles Werk mit bisher nicht publizierten Inhalten vor. Er schreibt nicht über die Ergebnisse seiner Tätigkeit, sondern informiert den Leser über die dahinterstehenden Motivationen. Der erste Teil wirkt Abschnittsweise etwas oberflächlich und - aufgrund der Aneinanderreihung mehrerer Kurzbiographien - hin und wieder langatmig. Mit dem zweiten Teil, und der im Zentrum stehenden Person von Carl O. Sauer, gewinnt die Publikation fortschreitend an Tiefe und gibt hervorragende Einblicke in die Entstehung und Entwicklung der von individueller Wissbegierde und romantischer Vorstellungskraft gekennzeichneten cultural-historical geography. Obwohl die enorme Bewunderung für den zweifellos einflussreichen Sauer teilweise etwas übertrieben wirkt, wird dessen große Bedeutung als Gründer und Identitätsstifter für die Mitglieder der Berkeley School - und mit ihr für einen gewichtigen Teil der US-amerikanischen Geographie - klar verständlich. Die ersten beiden Teile münden schließlich, in Form einer persönlichen Retrospektive und Introspektion Gades, in eine Synthese, die das bisher geschriebene in seiner Gesamtheit verständlich werden lässt; es ist vor allem die kontinuierliche Selbstbeobachtung - und Selbstkritik - des Autors, die es dem empathischen Leser ermöglicht in dessen Rolle zu schlüpfen. Besonders jüngeren, einer geisteswissenschaftlichen Geographie zugetanen Fachkollegen kann diese Publikation ein sehr hilfreicher Begleiter auf ihrer Entdeckungsreise zu den persönlichen Interessen sein - ganz im Sinne des zu Beginn erwähnten Zitats. 


\section{Literatur}

Borsdorf, A. und Leser, H.: Herbert Wilhelmy, DIE ERDE, 134, 14-15, 2003.

Leighly, J.: Berkeley. Drifting into geography in the twenties, Ann. Assoc. Am. Geogr., 69, 4-9, 1979.
Tuan, Y.-F.: Humanist Geography. An individual's search for meaning, George F. Thompson Publishing, Staunton, VA, USA, 2012. 